\title{
Review on Child Sexual Assault; influence of age on management strategies highlighted based on a case history
}

\author{
Vidanapathirana $\mathbf{M}^{1}$, Amararatne RRGS $^{2}$ \\ ${ }^{\prime}$ Department of Forensic Medicine, Faculty of Medical Sciences, University of Sri Jayewardenepura, Sri Lanka \\ ${ }^{2}$ Officer of the JMO, Teaching Hospital Colombo South, Kalubowila, Sri Lanka
}

Correspondence: Dr. M. Vidanapathirana

e-mail: mudithavidana@yahoo.co.uk

Key words: Child sexual assault, Contraceptive pills, STI prophylaxis, Counselling, Case conference, Hymenoplasty, Holistic management

\section{Introduction}

Medico-legal management of child survivours is entirely different from adults. However, in Sri Lanka, the management of child sexual assault is mainly conducted in order to satisfy the medico-legal objectives. Specialists in Forensic Medicine should work as a team with the other stakeholders in the management of child survivors of sexual assault for the best interest of the children while attending to medico-legal objectives. Holistic management (1) of child survivours of sexual assault is discussed in relation to a case presented to a forensic medical practitioner at a tertiary care hospital.

\section{Case report}

A 14-year old girl was alleged to have been sexually abused with ejaculation by a 16-year old boy. She felt severe pain and started bleeding from vagina and immediately informed her grandmother and then police.

Medico-legal examination was conducted 24 hours after the incident, after obtaining consent from grandmother. Her parents had left the family and she and her younger brother were living with grandparents. She had attained her menarche at the age of 13 and the last regular menstrual period was two weeks before. She was rational and oriented. Age appropriate secondary sexual characteristics were found.
There was a fresh complete hymenal tear at $6 \mathrm{O}^{\prime}$ clock position extending to the posterior fourchette indicating vaginal penetration. Samples were taken for further investigations. There was no evidence of anal penetration. She was referred to an obstetrician and emergency contraceptive pills were prescribed. She was treated at the mental health clinic. She did not suffer from sexually transmitted infections. A case conference was arranged and the child was handed over to her grandparents under the supervision of probation. Upon request of grandparents at the case conference, hymenoplasty was arranged.

\section{Discussion}

Sexual intercourse with a girl below 16 years is considered as statutory rape in Sri Lanka (2). Though there was evidence of statutory rape, the perpetrator being below 18 years he would be sent for rehabilitation for juvenile delinquency (3).

Emergency contraception can reduce the risk of pregnancy if used within 120 hours after unprotected intercourse and is most effective if used within 24 hours (4). When initiated within 72 hours, risk of pregnancy is reduced by $75 \%$ (4). The contraindications are the same as those used for ordinary contraceptive pills (5) and if LRMP is doubtful, pregnancy test should be performed before prescribing emergency contraceptive pills. In this case, the victim was referred to an obstetrician about 24 hours after the incident. Since the last regular menstrual period was 2 weeks before and the pregnancy test was negative, emergency contraceptive pills (ECP) were prescribed by the 
obstetrician. This reiterates the importance of offering ECP to prevent possible teenage pregnancies.

Eighty one percent of victims reported having psychological consequences after an incident of sexual assault (6). Psychological consequences may appear at different time intervals and mood changes and sleep disturbances were the most common (6). Prevalence of development of deliberate self-harm in survivours of sexual assaults is about $26 \%$ (7). This reiterates that the psychological symptoms or signs are not necessary to refer to the mental health expert. In this case, though there were no apparent psychological disturbances, routine psychiatric referral was done for counseling and management.

The overall prevalence of sexually transmitted infections (STI) among the survivours of sexual assault is about $26 \%$ (6) and routine referral is essential. Routine prophylactic therapy against STIs after a sexual assault is often recommended because follow up with these patients can be difficult and it also reduce the need for more expensive or extensive treatment if a STI is discovered at a later time (8). Evidence of STI is not essential in order to refer to the STI unit. Though the prophylaxis treatment of STI is not given to victims of sexual assault in Sri Lanka, it should be prescribed at least when there is evidence of penetration. In this case, though the child did not show evidence of STI, she was referred to the STI unit, and since she showed evidence of penetration, she was an eligible victim for prophylactic therapy.

The main objective of 'case conference' is not to find fault of anyone but to look into the welfare of the victim and the family (9). Though non-relative foster care services are not available in Sri Lanka, children are legally handed over to relatives under the supervision of the probation by the magistrate. Therefore, in this case, the conclusion of the case conference was to handover this child to grandmother under the supervision of the probation. This highlights the importance of arranging case conferences for the welfare of victims.

Sri Lankan culture is largely based on the values of the hymen. No vaginal bleeding at the honeymoon night is culturally considered as she has had prior sexual penetration with another man. Hymenoplasty is culturally sensitive and linked to a belief in certain cultures that the hymen is proof of virginity (10).
Hymenoplasty or hymen reconstruction surgery is a procedure to artificially restore the hymen and the aim is to reinstate bleeding during sexual intercourse, in particular for women who are getting married for the first time. Victims of statutory rapes, who receive hymenal damages by acts for which they cannot consent or comprehend, would be candidates for hymenoplasty.

In this case, after successful hymenoplasty surgery, the grandparents informed that they were greatly relieved and also changed the place of residence in order to prevent future social stigmas. In cases of statutory rape, after the medico-legal investigations, hymenoplasty should be available as an option whenever it is demanded.

Survivours of the sexual assault are re-victimized by different stakeholders of the management due to involvement and contact at different times. Joint Information Response Teams (JIRTs) comprised of community services, police and health professionals who undertake joint investigation of child protection matters, should be introduced to reduce revictimization for the best interest of children (11). Then, the survivors of the sexual assaults can be managed as 'whole' with minimal re-victimization. Further, by arranging case conferences, all the stake holders meet together and manage the victims in holistic manner considering the survivor and the family as a whole.

"National guidelines on examination for medicolegal purposes, reporting and management of sexually abused survivours" prepared by the College of Forensic Pathologists of Sri Lanka, "Sexual Assault Forensic Examination (SAFE)" programme conducted by USAID, and the "Guidelines for the multi-sectorial management of child abuse" prepared by the College of Paediatricians are prospective initiatives for the holistic management of survivours of sexual assault.

\section{References}

1. Ponti G, Tomasi A. Giuseppe Moscati (1880-1927): a holistic approach to medicine. Journal of Medical Biography 2013 Sep 16.

2. Section 363, rape, Penal code of Sri Lanka, department of government printing, Sri Lanka, 1970. 
3. Juvenile justice information portfolio: committee on rights of child, Sri Lanka, http://www.unicef-irc.org/ portfolios/documents/440_sri-lanka.htm (accessed 04.01.2013).

4. Gold MA. Emergency contraception: a second chance at preventing adolescent unintended pregnancy. Current Opinion in Pediatrics 1997; 9(4):300-9.

5. Committee on adolescence, Emergency contraception. Paediatrics 2012; 130(6): 1174-82.

6. Kawsar M, Anfield A, Walters E, McCabe S, Forster GE. Prevalence of sexually transmitted infections and mental health needs of female child and adolescent survivors of rape and sexual assault attending a specialist clinic. Sexually Transmitted Infection 2004; 80(2): 138-41.

7. Campbell L, Keegan A, Cybulska B, Forster G. Prevalence of mental health problems and deliberate self-harm in complainants of sexual violence. Journal of Forensic and Legal Medicine 2007; 14(2): 75-8.
8. Workowski KA, Levine, WC. Sexually Transmitted Diseases Treatment Guidelines, National Center for HIV, STD and TB Prevention, 2002, p.70.

9. Child Protection Case Conferences, Information for Practitioners, http://www.fifechildprotection.org.uk (accessed on 04.01.2013).

10. Hymenoplasty (Hymen Reconstruction) in Sri Lanka, http://www.health-tourism.com/hymenoplasty/sri-lanka (accessed 04.01.2013).

11. Joint information response team (JIRT), New South Wales, http://www.community.nsw.gov.au/welcome_to_docs_ website.html (accessed 04.01.2013). 